\title{
Maximum Tolerated Dose and Pharmacokinetics of Paclitaxel Micellar in Patients with Recurrent Malignant Solid Tumours: A Dose-Escalation Study
}

\author{
Olof Borgå · Roger Henriksson · Helena Bjermo · Elsa Lilienberg • \\ Nina Heldring $\cdot$ Niklas Loman
}

Received: January 18, 2019 / Published online: March 16, 2019

(C) The Author(s) 2019

\begin{abstract}
Introduction: A water-soluble Cremophor ELfree formulation of paclitaxel, in which retinoic acid derivates solubilize paclitaxel by forming micelles (paclitaxel micellar), was studied for the first time in man to establish the maximum tolerated dose (MTD) and to characterize the pharmacokinetics (PK).

Methods: This was an open-label, one-arm, dose-escalating study in patients with advanced solid malignant tumours, for which no standard therapy was available or had failed. Paclitaxel micellar was given as 1-h intravenous infusion every 21 days for 3 cycles, mainly without premedication. Plasma samples were collected
\end{abstract}

Enhanced digital features To view enhanced digital features for this article go to https://doi.org/10.6084/ m9.figshare.7707836.

O. Borgå

Borgå PK Consulting, Pär Lagerkvists gata 34, 11215

Stockholm, Sweden

R. Henriksson

Department of Radiation Sciences, Oncology, Umeå

University, 90187 Umeå, Sweden

H. Bjermo $\cdot$ E. Lilienberg · N. Heldring $(\varangle)$

Oasmia Pharmaceutical AB, Vallongatan 1, 75228

Uppsala, Sweden

e-mail: nina.heldring@oasmia.com

N. Loman

Department of Oncology, Lund University Hospital, Skåne University Hospital, 22185 Lund, Sweden during $24 \mathrm{~h}$ at the first cycle and paclitaxel concentrations were assayed by high-performance liquid chromatography. PK was evaluated using a two-compartment model.

Results: Thirty-four patients received paclitaxel micellar at doses ranging between 90 and $275 \mathrm{mg} / \mathrm{m}^{2}$. MTD was established as $250 \mathrm{mg} /$ $\mathrm{m}^{2}$. Fatigue and neuropathy were the most frequent dose-limiting toxicities. No hypersensitivity reactions were observed. PK of paclitaxel was evaluated in 25 data sets. Paclitaxel micellar had a rapid initial distribution phase, mean half-life $0.55 \mathrm{~h}$, estimated to be completed $3 \mathrm{~h}$ after dosing and a mean terminal half-life of $8.8 \mathrm{~h}$. Mean clearance was $13.4 \mathrm{~L} / \mathrm{h} / \mathrm{m}^{2}$ with fivefold interindividual variability. The residual areas after $10 \mathrm{~h}$ and $24 \mathrm{~h}$ were $15.7 \pm 8.6 \%$ and $5.7 \pm 3.9 \%$ of the area under the plasma concentration-time curve to infinite time $\left(\mathrm{AUC}_{\mathrm{inf}}\right)$, respectively.

Conclusion: No new side effects unknown for paclitaxel were observed. Maximum plasma concentration $\left(C_{\max }\right)$ and $\mathrm{AUC}_{\mathrm{inf}}$ showed a tendency to increase linearly with dose within the $150-275 \mathrm{mg} / \mathrm{m}^{2}$ dose range. The possibility to administer paclitaxel micellar without steroid premedication makes it an attractive candidate for further studies in combination with immunotherapy.

Trial Registration: EudraCT no: 2004-00182154.

Funding: Oasmia Pharmaceutical AB. 
Keywords: Cancer; Dose-finding; First-in-man; Nano-sized micelles; Paclitaxel micellar; Paclitaxel; XR17; Pharmacokinetics

\section{INTRODUCTION}

Paclitaxel plays a major role in treatment of ovarian cancer, lung cancer, and breast cancer, not only in the palliative but also in the adjuvant setting. Paclitaxel has a very low solubility in water, with an estimated value less than $0.1 \mu \mathrm{g} / \mathrm{mL}$ [1]. In the first marketed paclitaxel formulation, approved in the USA in 1992, a mixture of Cremophor EL and ethanol is used as solubilizer to enable parenteral administration of paclitaxel [2]. However, the use of Cremophor EL as a solubilizer can cause anaphylaxis and severe hypersensitivity reactions, such as dyspnoea, hypotension, angioedema and generalized urticaria, which occurred in $2-4 \%$ of patients receiving Cremophor EL formulated paclitaxel (Cremophor EL paclitaxel) in clinical trials [3]. Consequently, all patients receiving Cremophor EL paclitaxel should be pre-treated with corticosteroids and antihistamines $\left(\mathrm{H}_{1}\right.$ and $\mathrm{H}_{2}$ antagonists) and patients who experience severe hypersensitivity reactions to Cremophor EL paclitaxel should not be re-challenged with the drug [3]. Cremophor EL is also responsible for the non-linear pharmacokinetic (PK) behaviour of paclitaxel for Cremophor EL paclitaxel at clinical doses [4]. In addition, premedication with corticosteroids may potentially interfere with the immune response elicited by checkpoint inhibitors [5] and potentially affect efficacy in combination treatment including chemotherapy and immune therapy, suggesting that chemotherapy not requiring corticosteroid premedication may potentially be advantageous in that specific setting. Therefore, the main goals in the development of novel formulations of paclitaxel is to reduce toxicities associated with Cremophor EL and to decrease the need for premedication with corticosteroids $[6,7]$.

Extensive research has been performed to find competitive formulations to Cremophor EL paclitaxel and some of them have been more successful than others. For example, two of them have received market authorizations: nanoparticle albumin-bound paclitaxel in the USA (2005) and the European Union (2008) and paclitaxel micellar in the Russian Federation (2015) and European Union (2018) [8, 9]. In addition, several other formulations are in late stage clinical development such as nanoparticle polymer-based paclitaxel, liposomal paclitaxel, paclitaxel injection concentrate for nanodispersion (PICN) as well as nanosomal paclitaxel lipid suspension (NPLS) [6, 8].

Paclitaxel micellar is a Cremophor EL-free formulation of paclitaxel, in which two isoforms of $N$-retinoyl-L-cysteic acid methyl ester sodium salt (XR17) are used as micelle-forming excipients to make paclitaxel water soluble. When the lyophilized powder, containing paclitaxel and XR17, is dissolved in an aqueous medium, paclitaxel-containing micelles with sizes of $20-30 \mathrm{~nm}$ are formed. Paclitaxel micellar has been investigated in several clinical studies during the last decade. However, the results of the clinical trials have not been reported in any publication before.

The primary aim of this first-in-man study was to define the maximum tolerated dose (MTD) of paclitaxel micellar in patients with recurrent solid tumours. Secondary objectives were to describe the PK of increasing doses of paclitaxel when administered as this new micellar formulation.

\section{METHODS}

\section{Study Design}

This was an open-label dose-escalation study. Patients were enrolled at the Department of Oncology at Lund University Hospital and at Norrlands University Hospital, Umeå, Sweden, between November 2004 and May 2007. The protocol was approved by the Swedish Medical Products Agency (EudraCT number: 2004-001821-54) and the Regional Ethical Review Board in Lund, Sweden (Dnr 542/2004). The study was conducted in accordance with the protocol, regulatory requirements, Good Clinical Practice and the ethical principles of the Declaration of Helsinki. 
Male or female patients of at least 18 years old with a histologically proven solid malignant tumour for which no standard therapy was available or had failed were eligible. Other inclusion criteria were Eastern Cooperative Oncology Group (ECOG) performance status of up to 2 , and life expectancy of at least 12 weeks. Patients with significant abnormalities in blood chemistry, haematology or renal function, neuropathy (sensory or motor) of at least grade 2 , or autoimmune disease were not included.

\section{Drug Preparation}

Lyophilized powder of paclitaxel micellar (Apealea ${ }^{\circledR} /$ Paclical, Oasmia Pharmaceutical AB, Sweden) was dissolved in a sterile aqueous saline solution $(150 \mathrm{mmol} / \mathrm{L})$ with calcium chloride $(2.3 \mathrm{mmol} / \mathrm{L})$ for the first 9 patients, and in Ringer-acetate for injection for the remaining 25 patients to a concentration of $1 \mathrm{mg} / \mathrm{mL}$. The paclitaxel micellar solution was thereafter administered as a 1-h intravenous infusion for 3 cycles every 21 days.

\section{Dosing Procedure and Definition of MTD}

A stepwise dose escalation scheme was used starting at a dose of $90 \mathrm{mg} / \mathrm{m}^{2}$ (see Fig. 1). Briefly, the first two patients received increasing doses; thereafter, cohorts of three patients were included at each dose level for three 3-week cycles. If dose limiting toxicity (DLT) was recorded in one patient at one dose level, additionally three patients were treated at that same dose level. If at least two of the initial three patients or two of the six patients in the extended cohort experienced DLT, the MTD was defined as the dose level below that dose. Thereafter, six additional patients were administered the MTD for three treatment cycles. DLT was defined as one or more of the following adverse events (AEs): any life-threatening event possibly related to the study drug, any common grade 3 or 4 toxicity considered possibly related to the study drug, grade 4 neutropenia lasting for at least 7 days, grade 4 neutropenia with a fever over $38^{\circ} \mathrm{C}$, grade 3 thrombocytopenia lasting for at least 7 days, any grade 4 thrombocytopenia, grade 3 or worse non-haematological toxicity, except for nausea, vomiting and alopecia. Toxicity criteria were assessed according to National Cancer Institute's Common Terminology Criteria for Adverse Events (CTCAE) version 3.0.

Premedication with corticosteroids, $\mathrm{H}_{1}$ and $\mathrm{H}_{2}$ blockers was not given routinely but corticosteroids could be given at the discretion of the investigator. Patients were followed until 3 weeks after the last drug administration (end of trial visit at week 9), if not prematurely withdrawn.

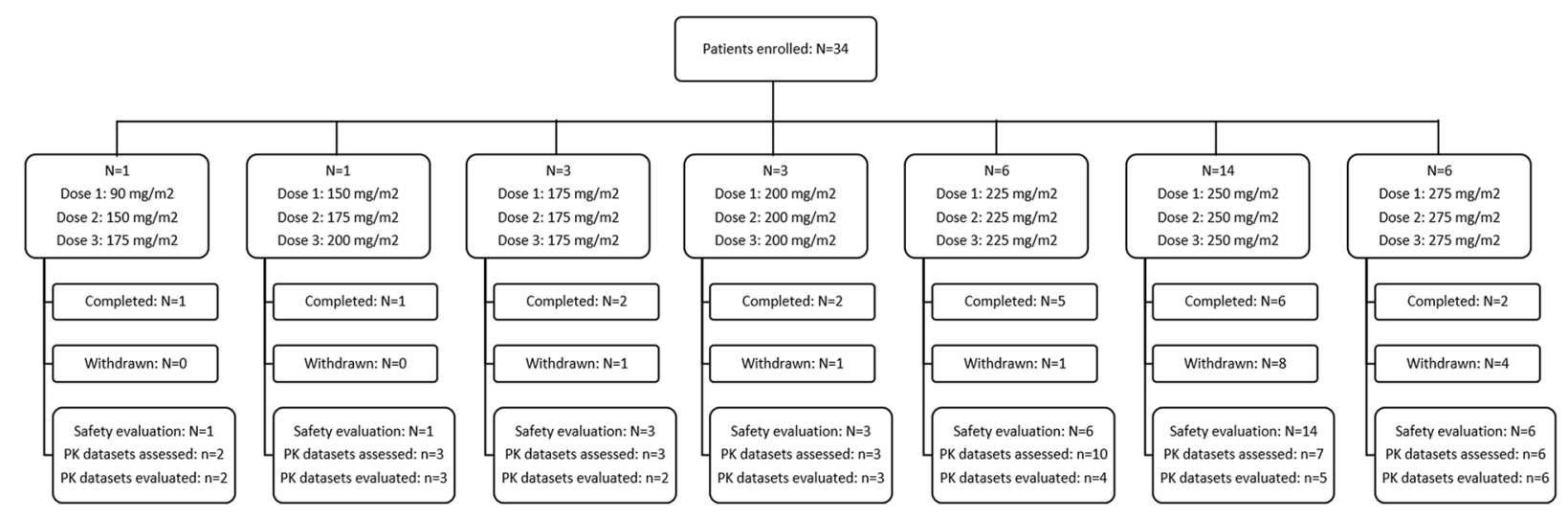

Fig. 1 Patient disposition. $N$ number of patients, $n$ number of data sets. Of the $34 \mathrm{PK}$ data sets assessed, nine were excluded from the PK evaluation because of the following reasons: missing actual sampling times $(n=1,175 \mathrm{mg} /$ $\mathrm{m}^{2}$ ), insufficient data for the PK model (i.e. only 2 post- infusion samples; $n=1,225 \mathrm{mg} / \mathrm{m}^{2}$ ), poor fit to the model $\left(n=2,225 \mathrm{mg} / \mathrm{m}^{2}\right.$, and $\left.n=2,250 \mathrm{mg} / \mathrm{m}^{2}\right)$ or when PK data sets were assessed repeatedly for the same patient at the same dose level $\left(n=3,225 \mathrm{mg} / \mathrm{m}^{2}\right)$ 


\section{PK of Paclitaxel}

\section{Blood Sampling}

Blood samples to determine plasma levels of paclitaxel were collected before infusion and at $5 \mathrm{~min}, 15 \mathrm{~min}, 30 \mathrm{~min}, 60 \mathrm{~min}, 3 \mathrm{~h}, 6 \mathrm{~h}, 8 \mathrm{~h}$, $10 \mathrm{~h}, 12 \mathrm{~h}$ and $24 \mathrm{~h}$ after start of infusion. Blood samples for PK analysis were taken during all 3 cycles in the first two patients, and in the first two patients who had been administered $225 \mathrm{mg} / \mathrm{m}^{2}$. For all other patients, samples were taken during the first treatment cycle only. The blood samples were collected in 5-mL sodiumheparin vacutainers, centrifuged and thereafter the plasma was transferred to cryovials and stored at $-70^{\circ} \mathrm{C}$ until analysis.

\section{Paclitaxel Concentration Analysis}

Paclitaxel concentrations in plasma were determined by Quintiles AB (Uppsala, Sweden) using a modification of a high-performance liquid chromatography (HPLC) method previously described by Sparreboom et al. [10]. In short, the assay consists of a rapid and highly selective liquid-liquid extraction step followed by HPLC using the mobile phases A) methanol/acetate buffer $\mathrm{pH}$ 5/tetrahydrofuran (40:60:2, v/v) and B) methanol/acetate buffer $\mathrm{pH} 5 /$ tetrahydrofuran (80:20:2, v/v). One-millilitre human plasma samples were spiked with internal standard (docetaxel) followed by extraction with $3 \mathrm{~mL}$ of acetonitrile/1-chlorobutane $(2: 8, \mathrm{v} / \mathrm{v})$ during 1 min on a Vortex mixer. The organic phase was separated and evaporated and the residue redissolved in mobile phase A before being injected onto the HPLC column. Isocratic elution with $36 \%$ mobile phase A and $64 \%$ mobile phase $B$ for $20 \mathrm{~min}$ (flow rate $0.5 \mathrm{~mL} / \mathrm{min}$ ) was used, at a column temperature of $60^{\circ} \mathrm{C}$. Detection was done with ultraviolet light at $230 \mathrm{~nm}$, and quantification was based on peak height ratios of paclitaxel to the internal standard. The calibration was obtained by a linear curve fit of the peak height ratios versus the concentration with a weighting factor of $1 /$ concentration. The method was validated in the range $10-500 \mathrm{ng} /$ $\mathrm{mL}$ with an inter-assay repeatability of $1.7-4.5 \%$ and an accuracy better than $4 \%$. The lower limit of quantification of paclitaxel in human plasma was $10 \mathrm{ng} / \mathrm{mL}$. It was ascertained that the XR17 component of the formulation did not interfere with the measurements.

\section{PK Data Analysis}

PK analysis was performed with individual plasma concentration-time data from each patient using WinNonlin PK software (Pharsight Corporation, Mountain View, CA, USA). In all PK calculations the actual given dose, infusion times and blood sampling times were used. If PK samples were collected for one patient at multiple cycles at the same dose level, the first evaluable data set was used in the PK evaluation. The data were found to be adequately described by a two-compartment model with a constant input rate during the infusion phase. All individual model-generated plasma concentration-time profiles were fitted to the input data by examining different weighting schemes and it was judged that a weight of $1 /\left(Y_{\text {hat }}\right)^{2}$ was most appropriate. If PK parameters could not be evaluated because of poor fits to the model (identified by visual inspection) these data sets were excluded from the evaluation. The following primary parameters were used to describe the two-compartment model: $V_{\mathrm{c}}, \mathrm{K} 10$, $\mathrm{K} 12$ and $\mathrm{K} 21$, where $V_{\mathrm{c}}$ is the apparent volume of the central compartment, K10 is the rate constant for elimination from the central compartment, $\mathrm{K} 12$ is the rate constant for drug transport from the central to the peripheral compartment and $\mathrm{K} 21$ is the rate constant for the opposite transport, i.e. from the peripheral back to the central compartment.

Secondary PK parameters obtained from the two-compartment model were the following: total area under the plasma concentration-time curve to infinite time $\left(\mathrm{AUC}_{\mathrm{inf}}\right)$, maximum plasma concentration $\left(C_{\max }\right)$, clearance $(\mathrm{CL})$, the distribution rate constant $\alpha$, the distribution half-life $\left(t_{1 / 2 \alpha}\right)$, the terminal elimination rate constant $\beta$, the terminal elimination half-life $\left(t_{1 / 2 \beta}\right)$, the distribution volume during the terminal elimination phase $\left(V_{\mathrm{z}}\right)$ and the distribution volume during steady state $\left(V_{\mathrm{ss}}\right)$. AUC $\mathrm{Anf}_{\text {inf }}$ was calculated from the following equation: 


$$
\mathrm{AUC}_{\mathrm{inf}}=\frac{\text { dose }}{K 10 \times V_{\mathrm{c}}}
$$

Note that these parameter values are based on the fitted model and therefore may differ numerically from traditional noncompartmental values, which are calculated directly from the raw data.

The rate at which the micelle formulation disintegrates in vivo was estimated with the assumption that the diameter of the micelles $(20-30 \mathrm{~nm})$ is too large to allow them to leave the blood intact by diffusion through pores in the capillary walls. The amount of drug in plasma during the infusion, when little drug has been eliminated from the body, will therefore to a large extent reflect the stability of the micelles. To characterize the micelle stability, the amount of drug in plasma at half the infusion time, $t_{\text {mid, }}$ was divided by the given dose, Dose $_{\text {mid, }}$ at the same time point according to the following equation:

$$
\begin{aligned}
& \text { Remaining dose fraction in plasma at } t_{\mathrm{mid}} \\
& =\frac{V_{\mathrm{p}} \times C_{\mathrm{p}, \mathrm{mid}}}{\text { Dose }_{\text {mid }}}
\end{aligned}
$$

where the amount of paclitaxel present in plasma is calculated from the plasma concentration at $t_{\mathrm{mid}}\left(C_{\mathrm{p}, \mathrm{mid}}\right)$, and the plasma volume $\left(V_{\mathrm{p}}\right)$ is approximated to be $1.7 \mathrm{~L} / \mathrm{m}^{2}$, assuming a total plasma volume of $3 \mathrm{~L}$ and a body surface area of $1.73 \mathrm{~m}^{2}$. Dose mid $_{\text {was }}$ estimated by the following equation:

$$
\text { Dose }_{\text {mid }}=\frac{\text { total dose } \times t_{\mathrm{mid}}}{T_{\mathrm{inf}}}
$$

where $T_{\text {inf }}$ is the total infusion time. When $T_{\text {inf }}$ deviated from $1 \mathrm{~h}$ and/or sampling time deviated from $0.5 \mathrm{~h}$, the sample taken closest to $T_{\text {inf }} /$ 2 was selected.

Since paclitaxel is available in plasma in both micellar and non-micellar form, this "remaining dose fraction in plasma" is the upper limit of the amount of paclitaxel in plasma that theoretically can be in its micellar form.

In order to characterize the contributed exposure over time, the areas under the concentration-time curve (AUC) during the first
$10 \mathrm{~h}\left(\mathrm{AUC}_{10 \mathrm{~h}}\right)$ or $24 \mathrm{~h}\left(\mathrm{AUC}_{24 \mathrm{~h}}\right)$ were calculated in each subject according to Eq. 4 :

$$
\mathrm{AUC}_{t}=\mathrm{AUC}_{\mathrm{inf}}-\frac{C_{t}}{\beta}
$$

where $\mathrm{AUC}_{t}$ is the AUC at time $t$ and $\mathrm{C}_{t}$ is the plasma concentration at time $t\left(C_{t} / \beta\right.$ corresponds to the residual area). The contribution of exposure over time was thereafter calculated as a percentage of $\mathrm{AUC}_{\text {inf }}$.

All data in the present study were summarized descriptively using STATA v 11 (STATA corporation).

\section{RESULTS}

\section{Patient Characteristics}

Thirty-four Caucasian patients (32-79 years) were included and received at least one dose of paclitaxel micellar. Nineteen patients (56\%) received all three treatment cycles.

Table 1 Characteristics of all patients and patients included in the PK evaluation

\begin{tabular}{lll}
\hline & $\begin{array}{c}\text { All patients } \\
(\boldsymbol{N}=\mathbf{3 4})\end{array}$ & $\begin{array}{l}\text { PK set } \\
(\boldsymbol{N}=\mathbf{2 2})\end{array}$ \\
\hline Sex (female/male) & $\begin{array}{c}15(44 \%) / 19 \\
(56 \%)\end{array}$ & $\begin{array}{c}8(36 \%) / 14 \\
(64 \%)\end{array}$ \\
Age (years) & $60 \pm 11$ & $62 \pm 10$ \\
Height $(\mathrm{cm})$ & $172 \pm 8$ & $173 \pm 8$ \\
Weight $(\mathrm{kg})$ & $73 \pm 14$ & $72 \pm 11$ \\
$\begin{array}{c}\text { Body surface area } \\
\left(\mathrm{m}^{2}\right)\end{array}$ & $1.9 \pm 0.2$ & $1.9 \pm 0.2$ \\
\end{tabular}

ECOG performance status

\begin{tabular}{lll}
0 & $9(26 \%)$ & $7(32 \%)$ \\
1 & $19(56 \%)$ & $12(55 \%)$ \\
2 & $6(18 \%)$ & $3(14 \%)$ \\
\hline
\end{tabular}

Data are presented as mean \pm standard deviation (continuous data) or counts and percentages (categorical data) ECOG Eastern Cooperative Oncology Group 
Table 2 Number of patients (\%) with serious adverse events listed by preferred term and dose of paclitaxel micellar

\begin{tabular}{|c|c|c|c|c|c|c|}
\hline \multirow[t]{2}{*}{ Preferred term } & \multicolumn{5}{|c|}{ Dose of paclitaxel micellar } & \multirow{2}{*}{$\begin{array}{l}\text { All patients } \\
(N=34)\end{array}$} \\
\hline & $\begin{array}{l}\overline{175 \mathrm{mg} / \mathrm{m}^{2}} \\
(N=5)^{\mathrm{a}}\end{array}$ & $\begin{array}{l}200 \mathrm{mg} / \mathrm{m}^{2} \\
(N=3)\end{array}$ & $\begin{array}{l}225 \mathrm{mg} / \mathrm{m}^{2} \\
(N=6)\end{array}$ & $\begin{array}{l}250 \mathrm{mg} / \mathrm{m}^{2} \\
(N=14)\end{array}$ & $\begin{array}{l}275 \mathrm{mg} / \mathrm{m}^{2} \\
(N=6)\end{array}$ & \\
\hline Ileus & $1(20 \%)$ & $1(33 \%)$ & & & & $2(6 \%)$ \\
\hline Subileus & $1(20 \%)$ & & & & & $1(3 \%)$ \\
\hline Stomatitis & & & & $1(7 \%)$ & & $1(3 \%)$ \\
\hline $\begin{array}{l}\text { Small intestinal } \\
\text { obstruction }\end{array}$ & & & & & $1(17 \%)$ & $1(3 \%)$ \\
\hline Pyrexia & $1(20 \%)$ & & $1(17 \%)$ & & $1(17 \%)$ & $3(9 \%)$ \\
\hline $\begin{array}{r}\text { Abdominal } \\
\text { infection }\end{array}$ & $1(20 \%)$ & & & & & $1(3 \%)$ \\
\hline Sepsis & & & & $1(7 \%)$ & & $1(3 \%)$ \\
\hline $\begin{array}{l}\text { Haemoglobin } \\
\text { decreased }\end{array}$ & & & & $1(7 \%)$ & $1(17 \%)$ & $2(6 \%)$ \\
\hline Arthralgia & & & & $1(7 \%)$ & & $1(3 \%)$ \\
\hline Myalgia & & & & $1(7 \%)$ & & $1(3 \%)$ \\
\hline Syncope & & & & $1(7 \%)$ & & $1(3 \%)$ \\
\hline Urinary retention & & & & $1(7 \%)$ & & $1(3 \%)$ \\
\hline Dyspnoea & & & & $1(7 \%)$ & & $1(3 \%)$ \\
\hline Thrombosis & & & & & $1(17 \%)$ & $1(3 \%)$ \\
\hline
\end{tabular}

${ }^{a}$ Data from the 2 patients receiving dose escalations are included at dose level $175 \mathrm{mg} / \mathrm{m}^{2}$

Demographic data for all included patients as well as for the patients included in the PK analysis are presented in Table 1 . The most common primary tumours were ovarian cancer $(N=6)$, malignant melanoma $(N=5)$, colon cancer $(N=4)$ and uterine cancer $(N=4)$. Patients were administered doses ranging between 90 and $275 \mathrm{mg} / \mathrm{m}^{2}$ as described in Fig. 1. Premedication was given before drug administration in four patients at 7 of 80 treatment cycles $(9 \%)$ in total.

\section{MTD and Safety}

Safety was evaluated in all 34 enrolled patients. DLTs occurred in three of six patients at a dose of $275 \mathrm{mg} / \mathrm{m}^{2}$ and MTD was established as $250 \mathrm{mg} / \mathrm{m}^{2}$. The MTD was validated in six patients. A total of 11 different preferred terms (PTs) were reported as 20 DLTs in eight patients. Fatigue and neuropathy (neuropathy, neuropathy peripheral or peripheral sensory neuropathy) were the most frequently occurring DLTs $(N=4[12 \%]$, respectively), followed by leukopenic events (leukopenia or febrile neutropenia)

Fig. 2 Plasma concentration-time curves for total paclitaxel at 1-h infusion of paclitaxel micellar at doses ranging between 90 and $275 \mathrm{mg} / \mathrm{m}^{2}$. a Mean concentrations for each dose level on a linear-linear scale, excluding the $90 \mathrm{mg}$ dose $(n=1)$ since it is outside the clinical dose range and mainly used to assess safety. $\mathbf{b}$ Individual (dots) and mean concentrations (continuous line) of data sets included in the PK evaluation, presented per dose level on log-linear scales. $n$ number of data sets 

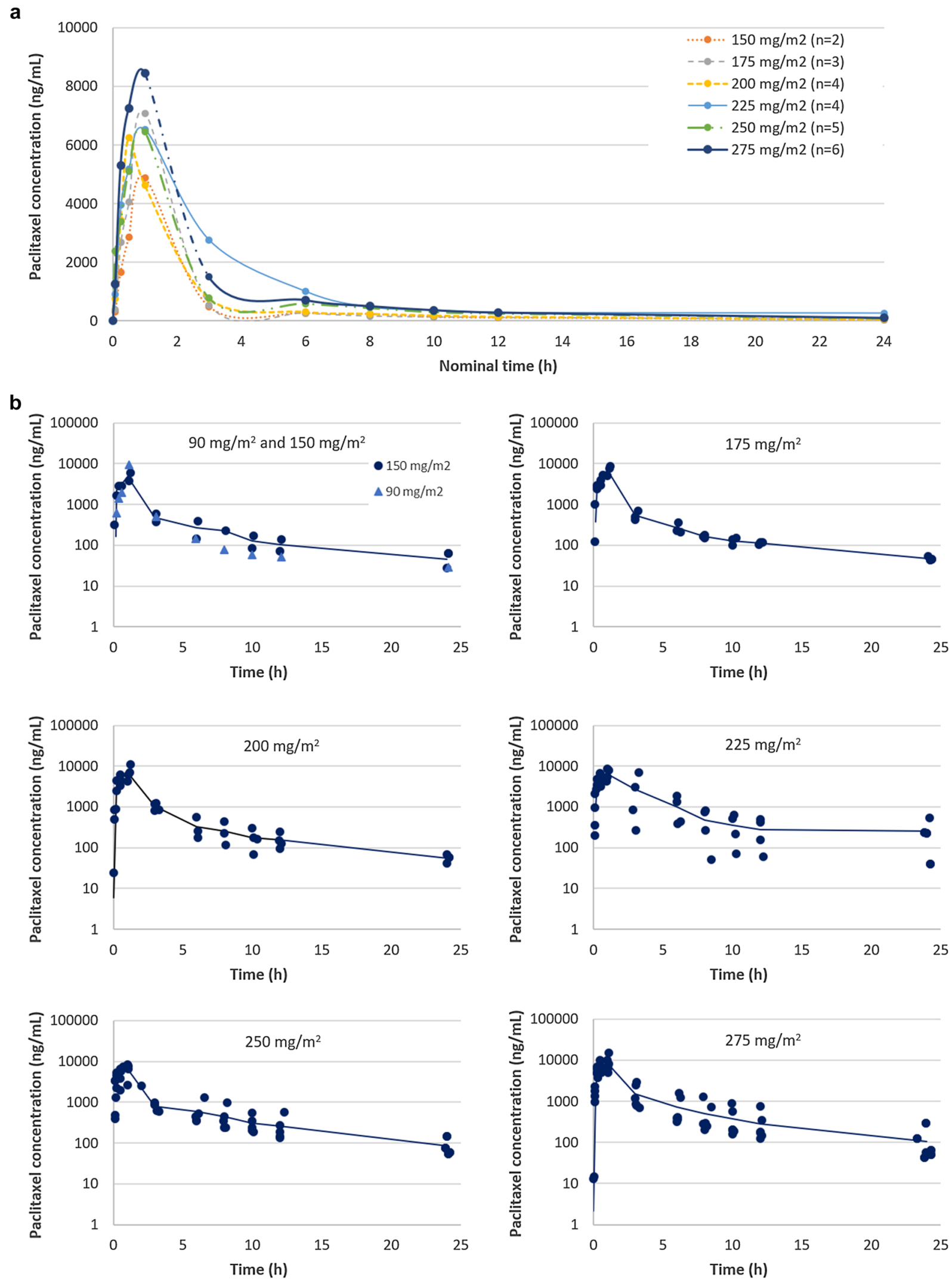


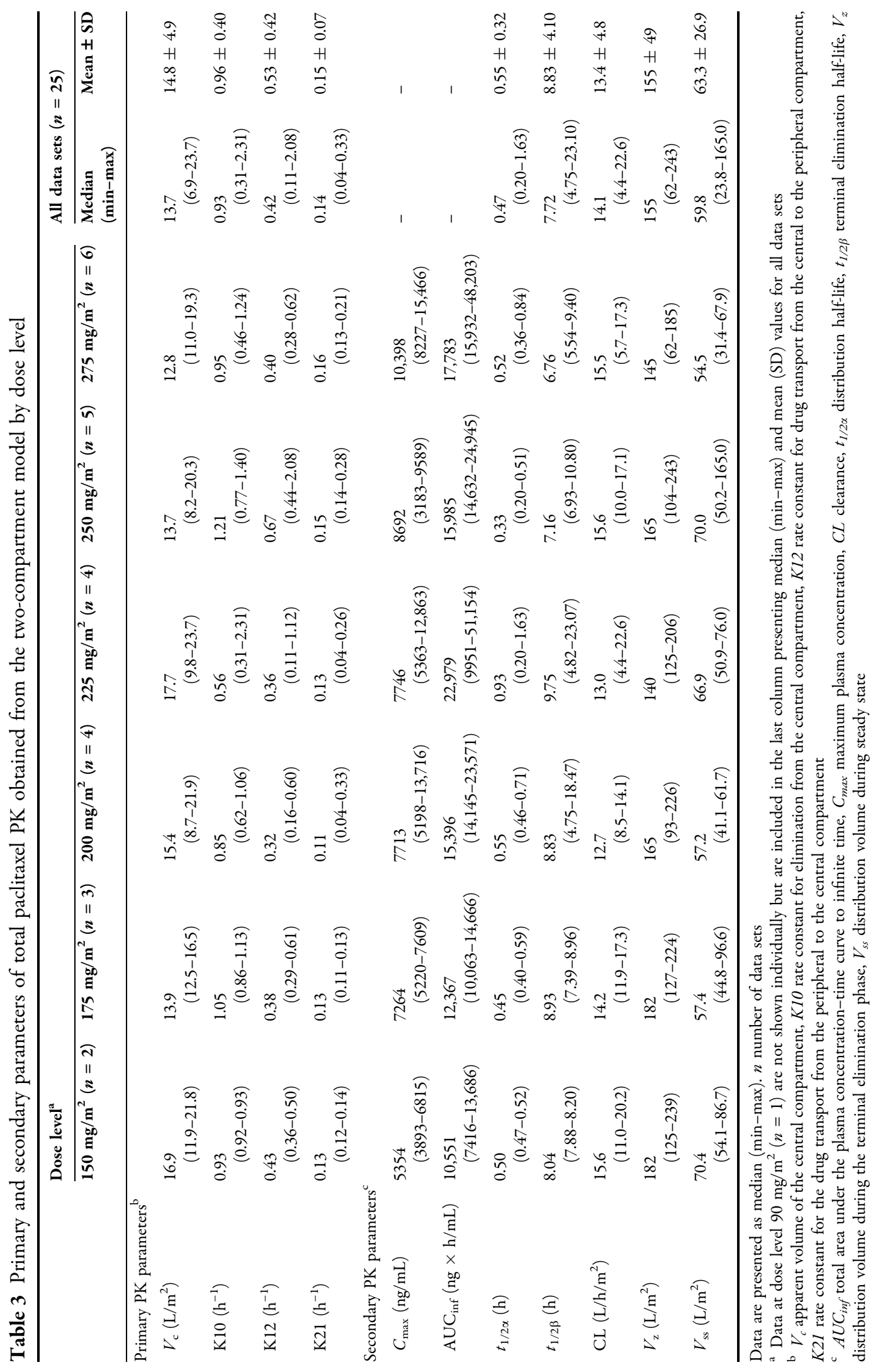


and stomatitis $(N=2[6 \%]$, respectively). The remaining DLTs occurred in single patients (small intestinal obstruction, arthralgia, myalgia, skin reaction). All DLTs occurred at paclitaxel doses of at least $225 \mathrm{mg} / \mathrm{m}^{2}$; one patient $(17 \%)$ at $225 \mathrm{mg} / \mathrm{m}^{2}$, four patients $(29 \%)$ at $250 \mathrm{mg} / \mathrm{m}^{2}$ and three patients (50\%) at $275 \mathrm{mg} /$ $\mathrm{m}^{2}$.

All 34 included patients experienced one or more $\mathrm{AE}$ and 12 patients (35\%) experienced a serious adverse event (SAE). The number of patients with SAE listed by preferred terms are presented in Table 2. There were no deaths during the study. Fifteen patients (44\%) discontinued the study after first or second treatment cycle, mainly because of AE with DLT and/or disease progression. Overall, the most common $\mathrm{AE}$ was fatigue $(N=23[68 \%])$. Other frequently occurring AEs were alopecia $(N=18$ [53\%]), leukopenia $(N=16[47 \%])$, neutropenia $(N=15[44 \%])$, peripheral sensory neuropathy (15 [44\%]), nausea (14 [41\%]) and pyrexia (13 [38\%]). AEs of neuropathy were reported in a total of 24 patients. Fourteen patients had a previous history of neuropathy and 11 of them experienced exacerbation or aggravation of such symptoms during treatment. No hypersensitivity reactions were observed relating to administration of paclitaxel micellar.

\section{PK of Paclitaxel}

\section{PK Evaluation}

A total of 34 sets of plasma concentration time data were obtained from 27 patients, at a total of seven dose levels $\left(90-275 \mathrm{mg} / \mathrm{m}^{2}\right)$. Nine data sets were excluded from the PK evaluation because of missing actual sampling time recordings $\left(n=1,175 \mathrm{mg} / \mathrm{m}^{2}\right)$, insufficient data for the PK model (i.e. only 2 post-infusion samples; $n=1,225 \mathrm{mg} / \mathrm{m}^{2}$ ), poor fit to the model $\left(n=2,225 \mathrm{mg} / \mathrm{m}^{2}\right.$, and $n=2,250 \mathrm{mg} /$ $\mathrm{m}^{2}$ ) or when PK data sets were repeatedly assessed for the same patient at the same dose level $\left(n=3,225 \mathrm{mg} / \mathrm{m}^{2}\right)$. The remaining 25 data sets were obtained from 22 subjects (see Figs. 1, 2). The $90 \mathrm{mg} / \mathrm{m}^{2}$ dose, which was outside the clinical dose range, was only administered to one single patient and mainly used to assess safety. Therefore, this dose level is only included in Fig. $2 \mathrm{~b}$ and in the dose-independent summary of PK parameters for all data sets (last column in Table 3). All infusions were administered during $0.95-1.15 \mathrm{~h}$, except on three occasions (1.30, 2.03, and $2.07 \mathrm{~h})$.

The plasma log concentration-time curves observed after a 1-h infusion of the paclitaxel micellar exhibited a biphasic shape (Fig. 2), consistent with the two-compartment model. The primary and secondary PK parameters obtained from the two-compartment model are presented in Table 3. The two-compartment analysis of paclitaxel in plasma demonstrated that the drug is rapidly leaving the central compartment, and distribution should be virtually complete $3 \mathrm{~h}$ after dosing, as the mean alpha half-life of paclitaxel was $0.55 \mathrm{~h}$.

The tissue distribution was extensive according to the large $V_{\mathrm{ss}}$, with a mean value of $63.3 \pm 26.9 \mathrm{~L} / \mathrm{m}^{2}$ or about $120 \mathrm{~L}$ for the average patient with a mean body surface area of $1.9 \mathrm{~m}^{2}$. The elimination half-life (beta half-life) varied from $4.75 \mathrm{~h}$ to $23.10 \mathrm{~h}$, with a mean value of $8.83 \pm 4.10 \mathrm{~h}$. CL varied from 4.4 to $22.6 \mathrm{~L} / \mathrm{h} /$ $\mathrm{m}^{2}$ with a mean value of $13.4 \pm 4.8 \mathrm{~L} / \mathrm{h} / \mathrm{m}^{2}$.

\section{Micelle Disintegration}

The remaining dose fraction of total paclitaxel present in plasma in the middle of the infusion interval was estimated in patients having received paclitaxel by a 1-h infusion (range $0.95-1.15 \mathrm{~h}$ ) at doses ranging from 90 to $275 \mathrm{mg} / \mathrm{m}^{2}$. The remaining dose fraction at mid-infusion was $7.8 \pm 2.1 \% \quad$ (range 4.6-13.2\%). This shows that persistent plasma levels of paclitaxel in its micellar form during the infusion are insignificant, indicating a rapid release from the formulation. There was no indication that this fraction was dosedependent.

\section{Dose Linearity}

Mean values of $C_{\max }$ and $\mathrm{AUC}_{\text {inf }}$ for the different dose groups (excluding the single subject that was studied at $90 \mathrm{mg} / \mathrm{m}^{2}$ ) were analysed by plotting them as a function of given dose and subjecting them to linear regression analysis (Fig. 3). $C_{\max }$ apparently increased in direct 

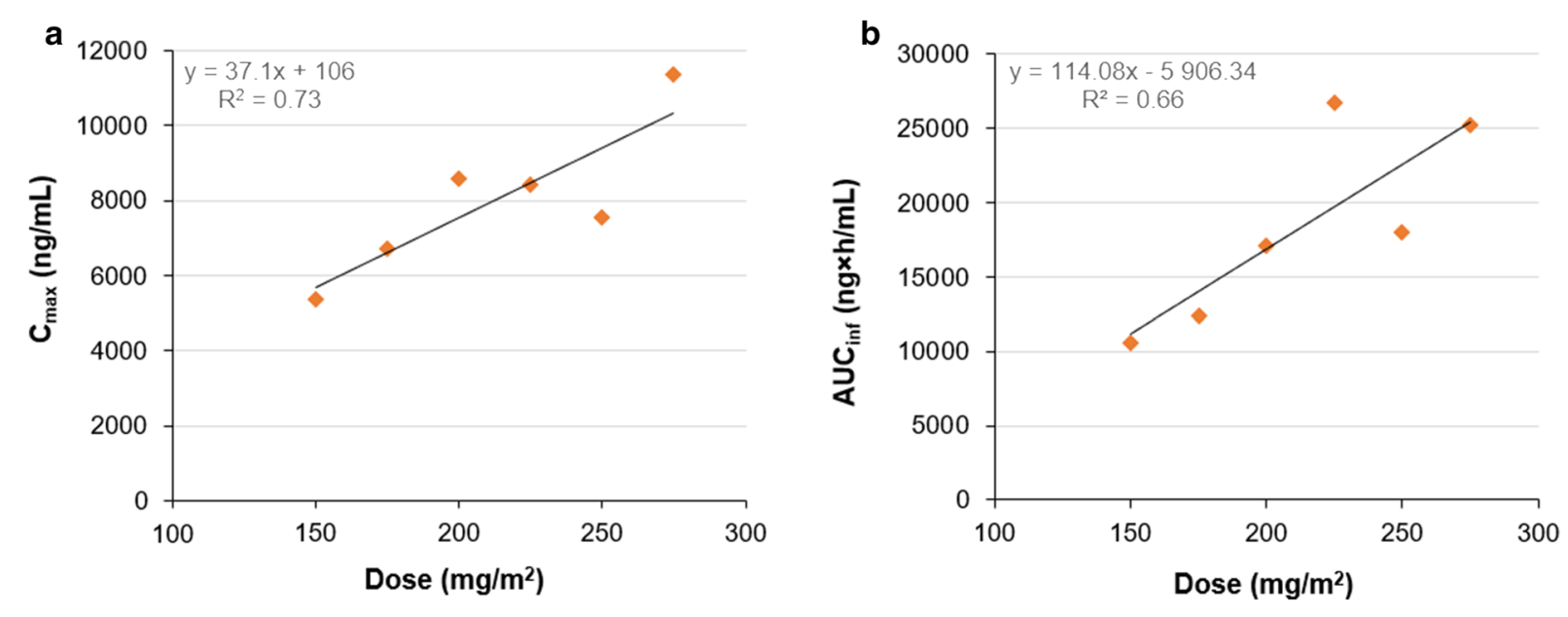

Fig. 3 Relationship between given dose and $\mathbf{a}$ mean $C_{\max }$ or $\mathbf{b}$ mean $\mathrm{AUC}_{\text {inf }}$ after a 1-h infusion for doses between $150 \mathrm{mg} /$ $\mathrm{m}^{2}$ and $275 \mathrm{mg} / \mathrm{m}^{2}$ of paclitaxel micellar

proportion to dose, although there was a considerable spread of the data points at the higher dose levels $\left(R^{2}=0.73\right)$ (Fig. 3a). In a similar way, $\mathrm{AUC}_{\text {inf }}$ was seemingly linearly correlated to dose $\left(R^{2}=0.66\right)$ (Fig. 3b).

\section{Estimations of Residual Area}

To characterize the generation of AUC with time, the residual area (the extrapolated AUC) was calculated in each subject using the 10 -h or 24-h sample as the last sampling point on the curve. These estimates showed that the majority of the total $\mathrm{AUC}_{\text {inf }}$ was generated during the first $10 \mathrm{~h}$, as the mean residual area ( $\mathrm{AUC}_{10 \mathrm{~h} \text {-inf }}$ ) accounted for only $15.7 \pm 8.6 \%$ of $\mathrm{AUC}_{\text {inf }}$ $(n=25)$. The mean residual area at $24 \mathrm{~h}$ accounted for $5.7 \pm 3.9 \%(n=23)$. Hence the area from $10 \mathrm{~h}$ to $24 \mathrm{~h}$ is only $10 \%$ of the total $\mathrm{AUC}_{\text {inf. }}$

\section{DISCUSSION}

This study was the first time the novel micellar formulation of paclitaxel was administered to man and the MTD was established as $250 \mathrm{mg} /$ $\mathrm{m}^{2}$. This is higher than the dose indicated for Cremophor EL paclitaxel [3], even if doses up to $250 \mathrm{mg} / \mathrm{m}^{2}$ have been administered [11]. A higher tolerated dose of paclitaxel formulated without Cremophor EL is in line with what previously has been established for nanoparticle albumin-bound paclitaxel (nab-paclitaxel), $260 \mathrm{mg} / \mathrm{m}^{2}$ [12], another Cremophor-free formulation of paclitaxel.

As a result of the absence of Cremophor, it was anticipated that hypersensitivity events would be less frequent with paclitaxel micellar. Therefore, no dexamethasone or antihistamine premedication was given in $90 \%$ of the treatment cycles. Exceptions were four patients who received premedication on the initiative of the investigator early in the study, since this was a first-in-man study. The most frequent AEs were in agreement with those reported in other studies for paclitaxel [3, 11-13], and no new side effects unknown for paclitaxel were observed. Fatigue and neuropathy were the most frequent DLTs. All neuropathies were experienced on dose level $225 \mathrm{mg} / \mathrm{m}^{2}$ or higher. Five patients had a grade 3 neuropathy (all at doses $250 \mathrm{mg} / \mathrm{m}^{2}$ or higher), of which all but one $\left(275 \mathrm{mg} / \mathrm{m}^{2}\right)$ had neurological symptoms already at inclusion. Haematological AEs were reported in 21 patients and were dose limiting in two patients (both on $250 \mathrm{mg} / \mathrm{m}^{2}$ dose level). Several patients experienced mild to moderate gastrointestinal tract symptoms (mucositis, nausea and diarrhoea) and gastrointestinal symptoms were classified as dose limiting in three patients.

The micellar formulation of paclitaxel was designed to make the lipophilic paclitaxel water soluble. The composition of the formulation 
does not indicate that it will act as a slow-release formulation of paclitaxel. However, there are no studies conducted on the stability of these paclitaxel-containing micelles in plasma. In the present study, the maximum amount of drug located in micelles in plasma during infusion was estimated to be only $7.8 \%$ (range 4.6-13.2\%), suggesting that the micelles rapidly disintegrate in vivo.

The $C_{\max }$ and $\mathrm{AUC}_{\text {inf }}$ tended to increase linearly with dose, although the data obtained in the present study are limited. In this context it is interesting to compare $C_{\max }$ of paclitaxel given as paclitaxel micellar with another rapidly dissolving formulation of paclitaxel, nab-paclitaxel. Data on mean $C_{\max }$ at different dose levels received during 0.5 -h infusions of nab-paclitaxel were extracted from a comprehensive review by Stage et al. [14] citing six studies and from a study by Nyman et al. [15] and plotted as a function of dose (Fig. 4). In order to make data from the present study comparable to those from the literature, individual $C_{\max }$ after $0.5-\mathrm{h}$ infusion of paclitaxel micellar was estimated by a simulation based on the two-compartment model. The estimated mean values of $C_{\max }$ after 0.5-h infusion of increasing doses of paclitaxel micellar are in line with data for nab-paclitaxel

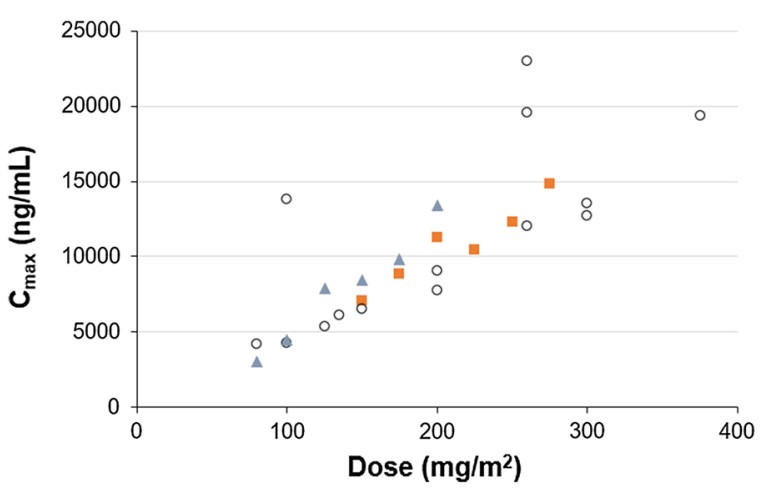

Fig. 4 Mean $C_{\max }$ of paclitaxel after 0.5 -h infusions of paclitaxel micellar (orange squares) and nab-paclitaxel (empty circles, blue triangles) at various dose levels. Nabpaclitaxel data were extracted from six studies compiled by Stage et al. (empty circles; [14]) and one study by Nyman et al. (blue triangles; [15]). Number of patients per dose level of paclitaxel micellar was as follows: $N=2(150 \mathrm{mg} /$ $\left.\mathrm{m}^{2}\right), N=3\left(175 \mathrm{mg} / \mathrm{m}^{2}\right), N=4\left(200 \mathrm{mg} / \mathrm{m}^{2}\right), N=4$ $\left(225 \mathrm{mg} / \mathrm{m}^{2}\right), N=5\left(250 \mathrm{mg} / \mathrm{m}^{2}\right), N=6\left(275 \mathrm{mg} / \mathrm{m}^{2}\right)$ in the $150-275 \mathrm{mg} / \mathrm{m}^{2}$ dose range (Fig. 4). In fact, Fig. 4 indicates a linear relation between dose and $C_{\max }$ for these two rapidly dissolving formulations of paclitaxel up to a dose of about $300 \mathrm{mg} / \mathrm{m}^{2}$.

The majority of the $\mathrm{AUC}_{\text {inf }}$, about $84 \%$, was generated during the first $10 \mathrm{~h}$. This number may be critical in designing future PK studies of paclitaxel micellar, as the European Medicines Agency (EMA) guideline on the investigation of bioequivalence requires that at least $80 \%$ of AUC is covered by the sampling scheme [16].

The mean CL values of paclitaxel when given as paclitaxel micellar in the present study should not be compared with the CL of paclitaxel given in Cremophor EL formulation. This is because the total paclitaxel, and not unbound paclitaxel, concentrations were measured in the present study. It has been reported that Cremophor EL paclitaxel has a non-linear PK which is explained by the fact that stable Cremophor EL-containing micelles are retained in plasma, in contrast to paclitaxel which is easily being distributed in the body. However, some paclitaxel will remain bound to the Cremophor EL micelles. Higher doses will generate higher plasma concentrations of Cremophor EL that will result in a higher proportion paclitaxel being bound to the Cremophor EL micelles [4]. This is equivalent to a lower apparent volume of distribution as well as a lower CL when total drug in plasma is measured, while PK parameters based on unbound drug levels will remain unaffected, according to a theory outlined for low clearance drugs like paclitaxel [17]. Total paclitaxel will appear to have non-linear PK, which is a mere consequence of the formulation rather than a true characteristic of paclitaxel itself [4]. Therefore, the true PK of paclitaxel in Cremophor EL formulations should be based on measurement of unbound drug concentrations in plasma [18]. However, total paclitaxel PK of Cremophor EL-free formulations should be comparable. The mean CL of paclitaxel including all dose levels in the present study was $13.4 \mathrm{~L} / \mathrm{h} / \mathrm{m}^{2}$. This can be compared with mean $\mathrm{CL}$ of nab-paclitaxel ranging from $11.9 \mathrm{~L} / \mathrm{h} / \mathrm{m}^{2}$ in four subjects receiving $375 \mathrm{mg} / \mathrm{m}^{2}$ [19] to $24.8 \mathrm{~L} / \mathrm{h} / \mathrm{m}^{2}$ in six subjects receiving $125 \mathrm{mg} / \mathrm{m}^{2}$ [20], and a majority in the range $20-25 \mathrm{~L} / \mathrm{h} / \mathrm{m}^{2}$ 
[14]. Mean CL of nab-paclitaxel tended to decrease at higher doses [14], which was not seen for paclitaxel micellar, probably as a result of the more limited dose range studied. There was a fivefold range, from 4.4 to $22.6 \mathrm{~L} / \mathrm{h} / \mathrm{m}^{2}$, in $\mathrm{CL}$ of paclitaxel micellar. Relatively few studies of nab-paclitaxel report on interindividual variability in $\mathrm{CL}$, but ranges between 8.8 and $16.7 \mathrm{~L} / \mathrm{h} / \mathrm{m}^{2}$ in 14 patients after doses of $250 \mathrm{mg} / \mathrm{m}^{2}$ [21] and between 8.7 and $43.4 \mathrm{~L} / \mathrm{h} /$ $\mathrm{m}^{2}$ in 14 patients receiving doses of $260 \mathrm{mg} / \mathrm{m}^{2}$ [22] have been reported. The latter variability is in line with that observed for paclitaxel micellar.

The present study was conducted in patients with metastatic malignant solid tumours for which they previously had been extensively treated. This contributes to a large interindividual variability, which further implicates some limitations for the study. The large interindividual variability prevents the establishment of dose linearity in the present study as the sample size within each dose level is probably too small to establish the true relationship between dose and $C_{\max }$ or $\mathrm{AUC}_{\text {inf }}$. Thus, the present study alone cannot be used to ascertain the dose linearity of paclitaxel micellar. However, the large variability in CL may be expected for paclitaxel as a combined consequence of interindividual differences in physiology, metabolic capacity and disease state of the patients. For example, it has been shown by simulations that polymorphic enzymes altering the metabolic capacity cannot be the sole explanation of this variability [23]. The physical status of the patients together with the small study population and short study duration also generate a lack of valid efficacy assessments.

Since the present first-in-man study was conducted, paclitaxel micellar has undergone later stages of clinical development. This includes a pivotal phase III study in platinumsensitive ovarian cancer patients where paclitaxel micellar was compared with Cremophor EL paclitaxel (both in combination with carboplatin), and a PK study comparing paclitaxel micellar with nab-paclitaxel. Paclitaxel micellar is now approved as an option to treat ovarian cancer patients in several markets.

\section{CONCLUSIONS}

This first-in-man study with the novel micellar formulation of paclitaxel established an MTD of $250 \mathrm{mg} / \mathrm{m}^{2} . C_{\max }$ and $\mathrm{AUC}_{\mathrm{inf}}$ of total paclitaxel tended to increase linearly with dose after 1-h infusions in the dose range $\left(150-275 \mathrm{mg} / \mathrm{m}^{2}\right)$. Simulation-derived estimates of $C_{\max }$ after $0.5-\mathrm{h}$ infusions showed approximately the same maximum levels for paclitaxel micellar as for nab-paclitaxel. In plasma, the micellar formulation appeared to disintegrate rapidly, the distribution phase that followed was short and the majority of $\mathrm{AUC}_{\text {inf }}$ was generated during the first $10 \mathrm{~h}$. Thus, paclitaxel has a rapid and extensive tissue distribution. In the studied dose range, there was no indication that $\mathrm{CL}$ decreased at higher doses. In agreement with previous data with nab-paclitaxel, interindividual variability in CL of paclitaxel micellar was also large. The fact that paclitaxel micellar safely can be given without premedication with corticosteroids makes it an attractive component in future studies combining chemotherapy with immune check-point inhibitors.

\section{ACKNOWLEDGEMENTS}

The authors would like to thank all participating patients and their families.

Funding. This study and the journal's article processing charges and Open Access fee were funded by Oasmia Pharmaceutical AB. All authors had full access to all of the data in this study and take complete responsibility for the integrity of the data and accuracy of the data analysis.

Authorship. All named authors meet the International Committee of Medical Journal Editors (ICMJE) criteria for authorship for this article, take responsibility for the integrity of the work as a whole, and have given their approval for this version to be published.

Disclosures. Olof Borgå is a salaried consultant at Oasmia Pharmaceutical AB. Roger 
Henriksson was an investigator in the clinical trial. Helena Bjermo is a full-time employee at Oasmia Pharmaceutical AB. Elsa Lilienberg is a full-time employee at Oasmia Pharmaceutical AB. Nina Heldring is a full-time employee at Oasmia Pharmaceutical AB. Niklas Loman was an investigator in the clinical trial.

Compliance with Ethics Guidelines. The protocol was approved by the Swedish Medical Products Agency (EudraCT number: 2004-001821-54) and the Regional Ethical Review Board in Lund, Sweden (Dnr 542/2004). All procedures performed in the study were in accordance with the protocol, regulatory requirements and Good Clinical Practice. They also adhered to the ethical standards of the institutional and/or national research committee and with the 1964 Helsinki declaration and its later amendments or comparable ethical standards. Informed consent was obtained from all individual participants included in the study.

Data Availability. The data sets generated during and/or analyzed during the current study are not publicly available due to confidentiality of Oasmia Pharmaceutical AB but are available from the corresponding author upon reasonable request.

Open Access. This article is distributed under the terms of the Creative Commons Attribution-NonCommercial 4.0 International License (http://creativecommons.org/licenses/ by-nc/4.0/), which permits any noncommercial use, distribution, and reproduction in any medium, provided you give appropriate credit to the original author(s) and the source, provide a link to the Creative Commons license, and indicate if changes were made.

\section{REFERENCES}

1. Konno T, Watanabe J, Ishihara K. Enhanced solubility of paclitaxel using water-soluble and biocompatible 2-methacryloyloxyethyl phosphorylcholine polymers. J Biomed Mater Res Part A. 2003;65(2):209-14.
2. Safavy A. Recent developments in taxane drug delivery. Curr Drug Deliv. 2008;5(1):42-54.

3. FDA, US Label. Taxol ${ }^{\circledR}$ (paclitaxel) injection (Patient Information Included). Bristol-Myers Squibb Company. 2011. Reference ID:2939751. http:// www.accessdata.fda.gov/drugsatfda_docs/label/ 2011/020262s049lbl.pdf. Accessed 8 Oct 2018.

4. Van Tellingen O, Huizing MT, Nannan Panday VR, Schellens JHM, Nooijen WJ, Beijnen JH. Cremophor EL causes (pseudo-) non-linear pharmacokinetics of paclitaxel in patients. $\mathrm{Br} \mathrm{J}$ Cancer. 1999;81(2):330-5.

5. Arbour KC, Mezquita L, Long N, et al. Impact of baseline steroids on efficacy of programmed cell death-1 and programmed death-ligand 1 blockade in patients with non-small-cell lung cancer. J Clin Oncol. 2018;36(28):2872-8.

6. Rajappa S, Joshi A, Doval DC, Batra U, Rajendranath R. Novel formulations of docetaxel, paclitaxel and doxorubicin in the management of metastatic breast cancer. Int J Nanomed. 2018;10:3757-69.

7. Kumar P, Raza K, Kaushik L, Malik R, Arora S, Prakash Katare $\mathrm{O}$. Role of colloidal drug delivery carriers in taxane-mediated chemotherapy: a review. Curr Pharm Des. 2016;22:5127-43.

8. Sofias AM, Dunne M, Storm G, Allen C. The battle of "nano" paclitaxel. Adv Drug Deliv Rev. 2017;122:20-30.

9. European Medicines Agency. Assessment report for Apealea. https://www.ema.europa.eu/documents/ assessment-report/apealea-epar-public-assessmentreport_en.pdf. Accessed 2 Feb 2019.

10. Sparreboom A, de Bruijn P, Nooter K, Loos WJ, Stoter G, Verweij J. Determination of paclitaxel in human plasma using single solvent extraction prior to isocratic reversed-phase high-performance liquid chromatography with ultraviolet detection. J Chromatogr B Biomed Sci Appl. 1998;705(1):159-64.

11. Omura GA, Brady MF, Look KY, et al. Phase III trial of paclitaxel at two dose levels, the higher dose accompanied by filgrastim at two dose levels in platinum-pretreated epithelial ovarian cancer: An intergroup study. J Clin Oncol. 2003;21(15):2843-8.

12. FDA, US Label. Highlights of prescribing information. Abraxane ${ }^{\circledR}$ for injectable suspension (paclitaxel protein-bound particles for injectable suspension). Celgene. 2018. Reference ID: 4307748. https://www.accessdata.fda.gov/ drugsatfda_docs/label/2018/021660s045lbl.pdf. Accessed 8 Oct 2018. 
13. Gradishar WJ, Tjulandin S, Davidson N, et al. Phase III trial of nanoparticle albumin-bound paclitaxel compared with polyethylated castor oil-based paclitaxel in women with breast cancer. J Clin Oncol. 2005;23(31):7794-803.

14. Stage TB, Bergmann TK, Kroetz DL. Clinical pharmacokinetics of paclitaxel monotherapy: an updated literature review. Clin Pharmacokinet. 2018;57(1):7-19.

15. Nyman DW, Campbell KJ, Hersh E, et al. Phase I and pharmacokinetics trial of ABI-007, a novel nanoparticle formulation of paclitaxel in patients with advanced nonhematologic malignancies. J Clin Oncol. 2005;23(31):7785-93.

16. European Medicines Agency. Guideline on the investigation of bioequivalence. CPMP/EWP/QWP/ 1401/98 Rev 1/Corr ${ }^{* *}$. 2010;1-27.

17. Rowland M, Tozer TN. Clinical pharmacokinetics and pharmacodynamics: concepts and applications. 3rd ed. Philadelphia: Wolters Kluwer Health/ Lippincott William \& Wilkins; 1995. pp. 164-165.

18. Brouwer E, Verweij J, De Bruijn P, et al. Measurement of fraction unbound paclitaxel in human plasma. Drug Metab Dispos. 2000;28(10):1141-5.
19. Ibrahim NK, Desai N, Legha S, et al. Phase I and pharmacokinetic study of ABI-007, a cremophorfree, protein-stabilized, nanoparticl formulation of paclitaxel. Clin Cancer Res. 2002;8(5):1038-44.

20. Ando M, Yonemori K, Katsumata N, et al. Phase I and pharmacokinetic study of nab-paclitaxel, nanoparticle albumin-bound paclitaxel, administered weekly to Japanese patients with solid tumors and metastatic breast cancer. Cancer Chemother Pharmacol. 2012;69(2):457-65.

21. Gardner ER, Dahut WL, Scripture CD, et al. Randomized crossover pharmacokinetic study of solvent-based paclitaxel and nab-paclitaxel. Clin Cancer Res. 2008;14(13):4200-5.

22. Sparreboom A, Scripture CD, Trieu V, et al. Comparative preclinical and clinical pharmacokinetics of a cremophor-free, nanoparticle albumin-bound paclitaxel (ABI-007) and paclitaxel formulated in cremophor (Taxol). Clin Cancer Res. 2005;11(11):4136-43.

23. De Graan AJM, Elens L, Smid M, et al. A pharmacogenetic predictive model for paclitaxel clearance based on the DMET platform. Clin Cancer Res. 2013;19(18):5210-7. 